\title{
Ultrasound Use in Urinary Stones: Adapting Old Technology for a Modern-Day Disease
}

\author{
David T. Tzou, MD, Manint Usawachintachit, MD,', Kazumi Taguchi, MD, PhD, ${ }^{1,3}$ and Thomas Chi, MD ${ }^{1}$
}

\begin{abstract}
Ultrasound has become a mainstay tool in urologists' armamentarium for the diagnosis and management of nephrolithiasis. From starting as a rudimentary form of imaging, it has come to play a more prominent role over time, paralleling evolution in ultrasound technology. Throughout the medical community there is a growing emphasis on reducing the amount of ionizing radiation delivered to patients during routine imaging. As such there has been a resurgence of interest in ultrasound given its lack of associated radiation exposure and proven effectiveness as a diagnostic and therapeutic imaging modality. Herein, we provide a review of the history of ultrasound, how the use of ultrasound is expanding in both diagnosis and treatment of urinary stone disease, and finally how promising applications of ultrasound are shaping the future of kidney stone management.
\end{abstract}

Keywords: extracorporeal shockwave lithotripsy, Image Guided Therapy, imaging, percutaneous nephrolithotomy, renal stone, ureteroscopy, urolithiasis

\section{Introduction}

$\mathbf{U}$ LTRASOUND HAS EVOLVED over the years from humble beginnings to its current role as a primary imaging modality for kidney stones. Initial efforts focused mostly on the development of ultrasound as a diagnostic imaging tool. Over time, however, new advances in ultrasound technology have facilitated its use as a therapeutic and therapy guidance aid. While there have been striking developments in ultrasound applications throughout all aspects of urology, this review article will focus on ultrasound with respect to the diagnosis, treatment, and management of nephrolithiasis.

\section{History of Ultrasound and Kidney Stones}

The use of ultrasound specifically for kidney stones dates back to 1961 when Schlegel and colleagues first reported on intraoperative amplitude (A)-mode ultrasonography for renal calculi. ${ }^{1}$ The image generated by this study was simply a single spike representing reflection from the renal calculus (Fig. 1).

Before ultrasound, kidney stones were diagnosed mainly using plain radiographs of the kidney, ureter, and bladder (KUB) along with intravenous pyeloureterograms. Given the inability of these tests to identify radiolucent stones, ultrasound soon emerged as a modality capable of identi- fying these kidney stones that were at the time difficult to visualize. Small case series by Edell and Zegel as well as Pollack and colleagues demonstrated the capabilities of grayscale ultrasound by showing how it could detect radiolucent uric acid and matrix stones, respectively, both of which were not appreciated on conventional plain-film urography (Fig. 2).,3

As ultrasound machines evolved, they became smaller, portable, and were soon capable of real-time imaging. This translated to the operating room as urologists started using ultrasound to facilitate stone localization and removal. Cook and Lytton were the first to describe intraoperative ultrasound using a brightness (B)-mode scanning probe to localize a stone and guide their nephrolithotomy. ${ }^{4}$

The focus of ultrasound has since shifted as the treatment options for nephrolithiasis evolved from open surgery via anatrophic nephrolithotomy to percutaneous nephrolithotomy (PCNL) and retrograde intra-renal surgery (RIRS). While ultrasound was previously used to identify stones to help guide the nephrolithotomy incision, ${ }^{4}$ it was soon applied to perform percutaneous access into the appropriate region of the collecting system for PCNL. ${ }^{5}$ Evolution in ultrasound machines has improved real-time image quality so that the modern day urologist has a distinct advantage compared to urologists of the past when it comes to characterizing a stone in vivo (Fig. 3).

\footnotetext{
${ }^{1}$ Department of Urology, University of California, San Francisco, San Francisco, California.

${ }^{2}$ Division of Urology, Faculty of Medicine, King Chulalongkorn Memorial Hospital, Chulalongkorn University, The Thai Red Cross Society, Bangkok, Thailand.

${ }^{3}$ Department of Nephro-urology, Nagoya City University Graduate School of Medical Sciences, Nagoya, Japan.
} 


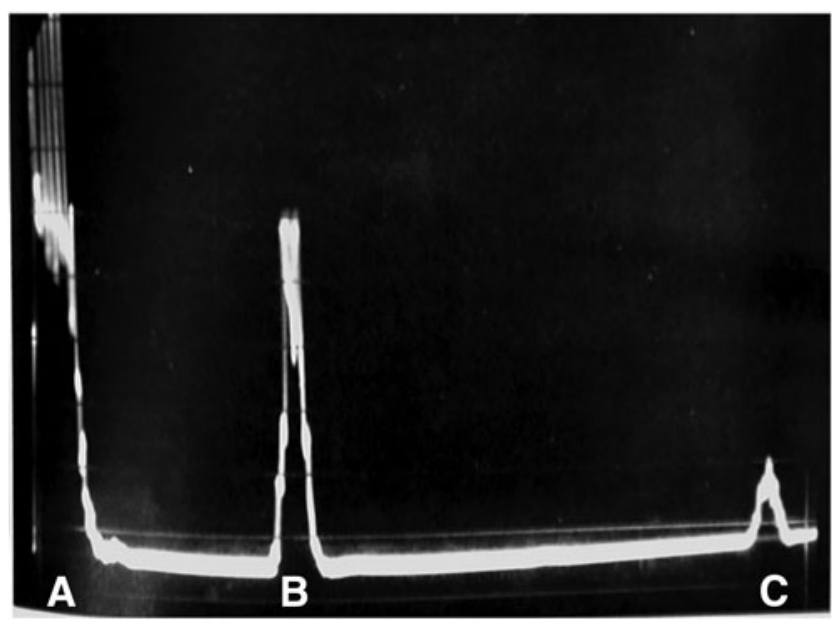

FIG. 1. A-mode sonogram of a renal calculus. The initial spike labeled (A) represents the surface of the kidney; the middle spike (B) represents a reflection from the calculus; finally the small last spike labeled $(\mathbf{C})$ represents the posterior margin of the kidney. Reproduced with permission from the Journal of Urology (see Schlegel et al.).

\section{Ultrasound as a Urolithiasis Diagnostic Modality}

\section{Acute stone episodes}

Given its high sensitivity and specificity, noncontrast CT (NCCT) scan became the widely accepted gold standard for diagnosing kidney stones by the end of the 20th century. This was accompanied with an explosion in the use of CT scans at the turn of the century to evaluate renal colic. Studies demonstrated that the number of annual CT scans increased from $\sim 3$ million per year in 1980, to over 62 million annually in 2006. ${ }^{6}$ Multiple studies have shown real risks associated with cumulative ionizing radiation exposure from repeat CT scans. As Ferrandino and colleagues demonstrated at two academic centers, $20 \%$ of patients received doses of radiation greater than the established International Commission on Radiation Protection occupational limit of $50 \mathrm{mSv}$ in a single year. ${ }^{7}$ One can imagine this likely underestimates the true extent of this overexposure phenomenon given that this study only tracked patients at two academic centers and did not account for those patients who had follow-up CT scans performed elsewhere. Throughout all medical specialties, there is a growing emphasis on limiting radiation exposure to "as low as reasonably achievable" (ALARA). As such there has been a resurgence in selecting ultrasound as the main initial imaging of choice in the diagnosis of nephrolithiasis.

This shift in imaging selection has been supported by studies comparing ultrasound with NCCT scans. These have shown that ultrasound can be an effective alternative and should be the preferred initial imaging study in the diagnosis of an acute stone episode. ${ }^{8} \mathrm{With}$ zero associated radiation and the ability to be performed quickly at the bedside, ultrasound has been demonstrated to help streamline the work-up of patients presenting to the emergency department (ED) with acute renal colic. ${ }^{9}$ A large, randomized, multicenter trial by Smith-Bindman and colleagues demonstrated that initial point-of-care ultrasound (POCUS) to evaluate patients with renal colic was associated with significantly lower 6-month cumulative radiation exposure compared to NCCT. ${ }^{10}$ POCUS had a sensitivity of $85 \%(95 \%$ CI 80,89$)$ compared to $86 \%$ (95\% CI 82, 90) for NCCT with respect to accurately diagnosing nephrolithiasis at 30-day follow-up. Moreover, there were no major differences between ultrasound and NCCT in return visits to the ED, subsequent complications, or number of serious adverse events (12.4\% POCUS vs $11.2 \%$ NCCT). Interestingly, POCUS was also associated with both a shorter ED length of stay compared to an ultrasound performed by a radiologist, and a lower total cost compared to NCCT. ${ }^{10}$ Reflecting these findings, ultrasound is now considered by many to be the initial imaging test of choice to evaluate patients with suspected nephrolithiasis. Per European Association of Urology (EAU) 2016 Guidelines: "ultrasound should be used as the primary diagnostic imaging tool before performing a NCCT scan for stone confirmation.",11

\section{Pediatric and pregnant patients}

For pediatric and pregnant patients, ultrasound is widely accepted as the standard initial diagnostic imaging modality for both of these vulnerable patient populations. ${ }^{12,13}$ For pediatric patients, previous reports have demonstrated ultrasound to have $70 \%$ sensitivity, $100 \%$ specificity, $96 \%$ positive predictive value, and $62 \%$ negative predictive value to identify a renal stone. ${ }^{14}$ Ultrasound has a decreased sensitivity in pregnant patients, estimated to be $\sim 34 \%$, given that the physiologic changes of pregnancy make it difficult to discern physiologic ureteral dilation from obstruction due to a ureteral stone. ${ }^{15}$ However, these are two patient populations
FIG. 2. Early examples of ultrasound detecting radiolucent calculi. (A) Prone longitudinal section $9 \mathrm{~cm}$ to the left of the midline shows a dense collection of echoes with a marked acoustic shadow (arrow), indicating a left renal calculus. Reproduced with permission from Radiology (see Pollack et al.). (B) Longitudinal renal echogram demonstrating sonic shadows (arrows). Reproduced with permission from American Journal of Roentgenology (see Edell and Zegel).
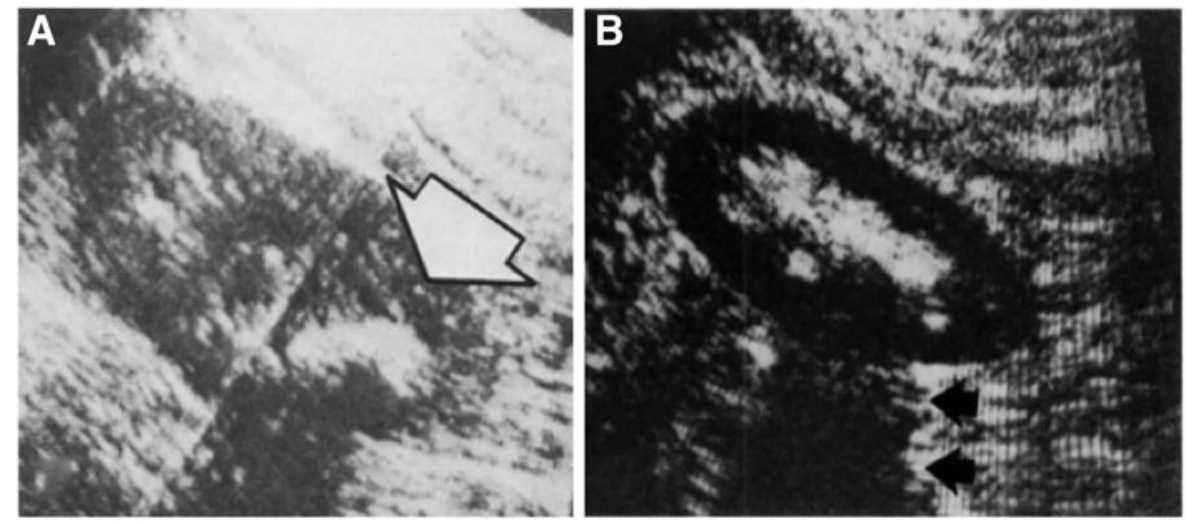

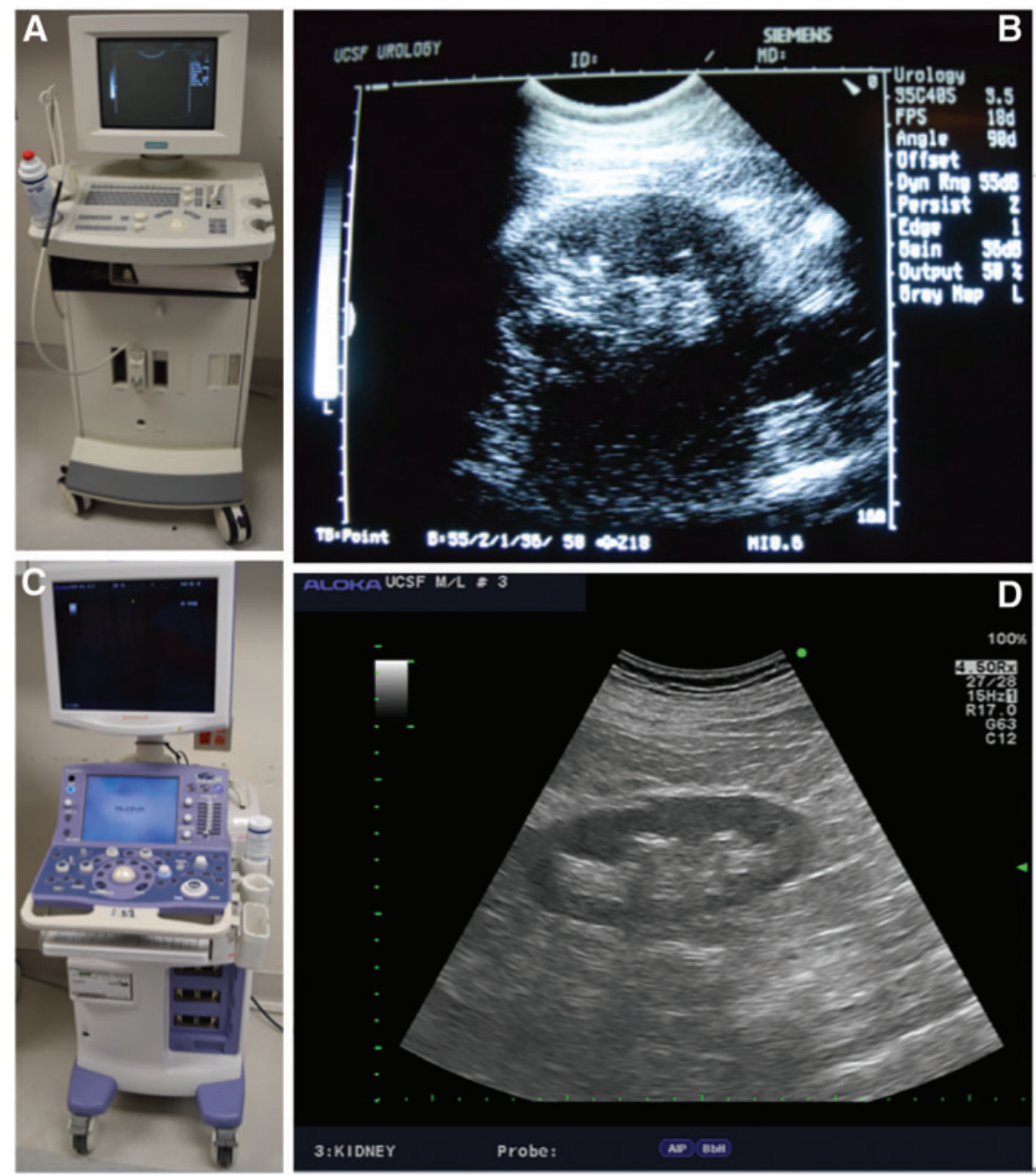

FIG. 3. Comparison between older and contemporary ultrasound imaging consoles. Notice differences in console size and shape as well as image quality. (A) Siemens Sonoline ${ }^{\circledR}$ Prima ultrasound unit from 1995. (B) Longitudinal image of a normal kidney with Siemens unit. (C) Hitachi Aloka ProSound Alpha 7 ultrasound console currently in production. (D) Longitudinal image of a normal kidney with Hitachi unit. for whom exposure to ionizing radiation is particularly detrimental. Given this serious concern, ultrasound remains a primary diagnostic imaging modality to evaluate for the presence of urolithiasis. In each of these patient populations, both American Urological Association (AUA) and EAU guidelines recommend performing ultrasound first, followed by MRI in pregnant patients and low-dose CT scans in pediatric patients to evaluate for urinary stones. ${ }^{11,16}$

\section{Advances in diagnostic ultrasound}

While there has been a conscious effort to limit NCCT radiation exposure with the recent advent of low-dose and very low-dose scans, ${ }^{17}$ one question that arises is whether radiation exposure is needed at all for the management of an acute stone episode. The reasoning underlying this question is whether the anatomic detail provided by these studies is worth their higher radiation exposure. Recent advances in the technical aspects of ultrasound may be providing an answer. The use of twinkling Doppler ultrasound has shown promise as an imaging modality to replace NCCT scans for identifying ureteral stones. ${ }^{18}$ Abdel-Gawad and colleagues demonstrated in a prospective, radiologist blinded study of 815 patients that color Doppler ultrasound (CDU) was able to identify $97.1 \%$ of patients with ureteral stones, with an associated $97.2 \%$ sensitivity and $99 \%$ specificity. ${ }^{19}$ A strength of this study was that multiple ultrasound machines were each able to reproduce the twinkling effect. These results may have been limited by an absence of evaluation of intra- and inter-observer variability, and these imaging studies require expertise in performing both graded compression of the ureter and interpreting this novel technique. However, such findings suggest that with further development, CDU could provide imaging accuracy to compete with NCCT.

Previous studies have demonstrated that ultrasound can overestimate urinary stone size, especially with regard to small stones. ${ }^{20}$ Recent studies have shown that this discrepancy can be secondary to increased depth and gain on commercial ultrasound machines. ${ }^{21}$ Dunmire and colleagues demonstrated that stone size accuracy improved significantly by measuring acoustic shadow width instead of stone width. $^{22}$ Furthermore, a study by May and colleagues described the accuracy of stone-specific algorithms (S-mode) to help estimate kidney stone size in vivo, demonstrating size estimation within $1 \mathrm{~mm}$ of stone size on NCCT. ${ }^{23}$ These studies illustrate encouraging progress in improving the diagnostic capabilities of ultrasound for the accurate detection of kidney stones. 


\section{Surveillance imaging}

Given that up to $50 \%$ of patients who experience a kidney stone will have a recurrent stone episode within 5 years, regular surveillance is recommended. ${ }^{24}$ With an effective dose for a KUB being 0.2 to $0.7 \mathrm{mSv}$ compared to 4.3 to $6.5 \mathrm{mSv}$ for a single-detector NCCT, most urologists have adopted a strategy of combining renal ultrasound with a KUB in an effort to minimize cumulative radiation exposure. ${ }^{25}$ Moreover, prospective studies have demonstrated that a KUB and ultrasound combination can have similar sensitivity and specificity compared to NCCT. ${ }^{26}$ Asymptomatic patients are usually imaged on a yearly basis but a schedule is often tailored for each individual. With no consensus guidelines on this schedule, future research is needed to determine the optimal imaging surveillance strategy for patients with a history of urinary stone disease.

\section{Use of Ultrasound in Urinary Stone Treatment}

\section{Ultrasound-guided access for PCNL}

Since being described by Wickham and colleagues in 1981, fluoroscopy has been the main imaging modality to guide percutaneous access into the collecting system. ${ }^{27}$ However, fluoroscopy has associated disadvantages; it does not allow clear identification of visceral organs around the kidney and it can be difficult to readily identify the posterior calyx of choice for collecting system entry. In addition, the associated radiation exposure to the patient, treating surgeon, and operating room staff has always been a potential source of negative consequences. Studies focused on effective radiation dose from PCNL have found that as body mass index, stone burden, nonbranched stone configuration, and number of access tracts increases, so does radiation exposure. ${ }^{28}$ Organ-specific radiation doses have also been estimated for PCNL and shown to have a nonuniform dose distribution. ${ }^{29}$ With published fluoroscopic series generally demonstrating a screening time of more than 200 seconds, ${ }^{28}$ alternative strategies for decreasing and eliminating the use of fluoroscopy from PCNL are appealing for the practicing urologist.

Desai and colleagues in 1999 were the first to report ultrasound-guided PCNL with 45 procedures performed in pediatric patients. ${ }^{5}$ Since the advent of the 21 st century, multiple studies have looked at the effectiveness of ultrasound and compared its safety to that of fluoroscopy. In a randomized prospective trial of 224 patients undergoing PCNL, Agarwal and colleagues demonstrated that compared to fluoroscopy guidance, ultrasound-guided access resulted in higher puncture accuracy, fewer puncture attempts, lower radiation exposure, and shorter operative times, all while maintaining similar stone-free rates and length of hospital stay. ${ }^{30}$ Other large series of ultrasound PCNL have described high clinical stone-free rates of $95.4 \%$ for single stones and $74.8 \%$ for multiple/staghorn stones. ${ }^{31}$ Ultrasound-guided access for PCNL appears to reduce intraoperative radiation exposure while maintaining safe clinical outcomes.

While renal access remains the most crucial aspect of performing PCNL, investigators have extended the use of ultrasound to include all steps of the operation. Some have reported using ultrasound guidance to position a fascial dilator, but then a two-step technique to complete tract dilation requiring a small sized scope to confirm proper tract location. ${ }^{31}$ Our group has shown that every step of PCNL - from renal access to tract dilation using a balloon dilator to nephrostomy tube placement - can be performed with ultrasound to directly visualize and guide each step. (Fig. 4). ${ }^{32}$ Encouragingly this technique can be applied successfully even in obese patients ${ }^{33}$ and adopted relatively quickly with a learning curve of less than 20 procedures. ${ }^{34}$ Moreover, obese patients may benefit the most from ultrasound guidance as the same fluoroscopic screening time has been shown to result in higher radiation exposure in obese $v s$ nonobese patients. ${ }^{33}$

As the use of ultrasound for PCNL continues to grow, it is reasonable to expect the development of higher resolution real-time imaging, fusion, and other technologies will improve visualization for renal access. Moreover dilators, balloon dilation devices, and nephrostomy tubes composed of proper acoustic surfaces would all contribute to providing better visualization and making it easier to perform X-rayfree ultrasound PCNL.

\section{Ultrasound fusion imaging}

Guidance positioning system (SonixGPS ${ }^{\mathrm{TM}}$; Ultrasonic Medical Corporation) is an ultrasound-guided needle tracking system that provides the current and predicted needle tip intracorporeal position in a real-time manner. This system uses multiple position electromagnetic sensors, including one embedded in the ultrasound transducer and another in the GPS needle, for delivering an accurate needle position. The operating surgeon can monitor the needle tip readily even when it is not in-plane with the ultrasound image or even when it is deeply located in tissue. ${ }^{35} \mathrm{Li}$ and colleagues have reported a total of 97 patients randomized into SonixGPS and conventional ultrasound guidance. While preoperative parameters and stone-free rate were comparable, time to successful puncture (7.7 vs 12.4 minutes), number of trials for successful puncture (1.3 vs 3.2), operative time, hospital stay, and change in hemoglobin were all significantly decreased in the SonixGPS
FIG. 4. Example of intraoperative ultrasound being used for balloon dilation during PCNL. (A) The nondominant hand holds the ultrasound probe while the dilator is advanced with the dominant hand to maintain live imaging of the dilation tract. (B) Ultrasound image demonstrating balloon dilator (arrow) advancing into position within the kidney. PCNL= percutaneous nephrolithotomy.
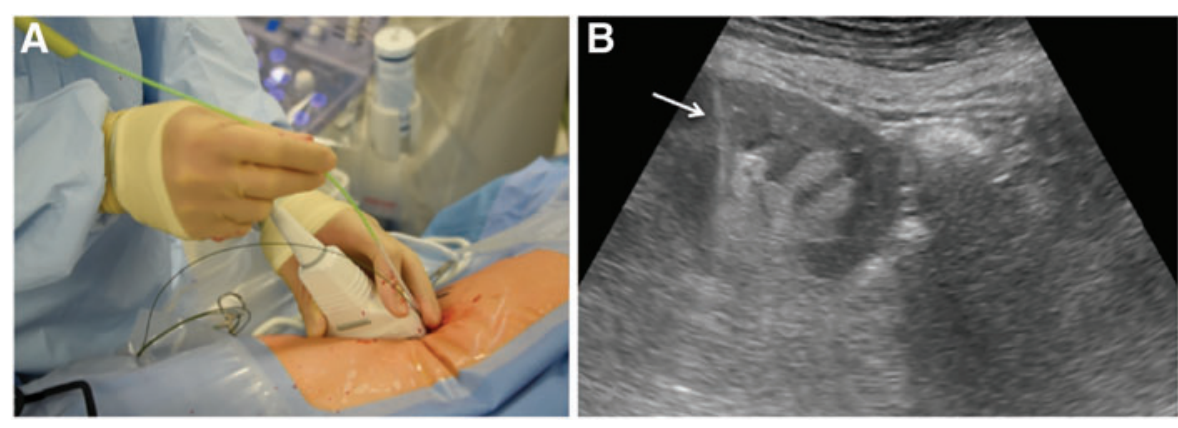
group. ${ }^{35}$ With the efficacy and safety of this technique confirmed in patients with complex renal stones, ${ }^{36}$ this system represents a promising new technology for use in PCNL.

\section{Ultrasound use in RIRS}

Though RIRS has traditionally relied on fluoroscopic guidance, there are potential benefits to performing ureteroscopy using ultrasound guidance. Besides being free of radiation, ultrasound allows for continuous intraoperative monitoring of the collecting system with unlimited real-time verification of guidewire, scope, and stent placement. Deters and colleagues used intraoperative ultrasound to guide flexible ureteroscopy in seven pregnant patients with suspected urolithiasis at a gestational age between 13 and 33 weeks. ${ }^{37}$ This technique served to confirm guidewire position within the renal pelvis at the beginning of the procedure, identify all calices during stone fragmentation, and confirm stent position within the renal pelvis at the end of the operation. A subsequent randomized controlled trial compared ultrasound vs fluoroscopy-guided ureteroscopy for 50 symptomatic patients with small ureteral stone patients, all of whom were pre-stented. ${ }^{38}$ No significant differences were found in either mean operative times (ultrasound: 36.5 minutes vs fluoroscopic: 45.7 minutes) or stone-free rates (both equal at $86 \%$ ). While each treatment group experienced a few minor postoperative complications, rates were not significantly different between the two groups.

There are some limitations for ultrasound-guided RIRS. It usually requires a second surgeon or a sonographer to control an ultrasound probe and identify the kidney in a real-time manner. Visualization of a ureteral access sheath can be challenging and ultrasound imaging may be limited in obese patients or those with a contracted body habitus. With technological advancements, future studies may be able to address these areas.

\section{Ultrasonic propulsion}

Investigators have shown the ability of transcutaneous ultrasonic propulsion to help reposition stones and potentially facilitate their passage in human subjects. ${ }^{39}$ Harper and colleagues investigated the use of a diagnostic ultrasound device with the ability to provide focused higher amplitude, longer duration pulses (VDAS; Verasonics ${ }^{\circledR}$ ) with either a low $(50 \mathrm{~V})$ or high $(90 \mathrm{~V})$ push voltage. ${ }^{39}$ With a limited number of patients, this study showed that ultrasonic propulsion can move smaller sized stones and also demonstrated a diagnostic ability to differentiate a cluster of small passable stones from what was perceived to be a single larger stone. This promising technology may provide novel therapeutic and diagnostic capabilities.

\section{Ultrasound for extracorporeal shockwave lithotripsy}

One of the challenges encountered during extracorporeal shockwave lithotripsy (SWL) is maintaining the energy focus on a stone given that it moves in accordance with the patient's respiration. For most lithotripters, usually less than half of the shockwaves are accurately focused on the stone, and therefore the renal parenchyma and other organs can be damaged by this misdirected energy. ${ }^{40}$ The LM-9200 ELMA (LiteMed) lithotripter has been shown to provide real-time outline tracking that resulted in reduced number of shockwaves and shorter treatment times for SWL. ${ }^{41}$ Meanwhile Visio-Track (VT) (EDAP-TMS) is a free-line three-dimensional ultrasound stone locking system that also provides real-time monitoring and has been shown to result in significantly less fluoroscopic time and radiation of exposure. ${ }^{42}$ Incorporating ultrasound for SWL appears to be the next step in the evolution of this wellestablished treatment modality.

\section{Conclusions}

There has been resurgence in the use of ultrasound for both diagnosis and treatment of nephrolithiasis. As the field of urology moves toward decreasing the amount of radiation exposure from CT scans and fluoroscopy, ultrasound will play an increasingly prominent role for the modern day urologist. Ultrasound technology has made significant advancements during the last several decades and this trend will likely continue to make ultrasound even more facile over time.

\section{Acknowledgment}

This study was supported by K12-DK-07-006: Multidisciplinary K12 Urologic Research Career Development Program (T.C.).

\section{Author Disclosure Statement}

No competing financial interests exist.

\section{References}

1. Schlegel JU, Diggdon P, Cuellar J. The use of ultrasound for localizing renal calculi. J Urol 1961;86:367-369.

2. Pollack HM, Arger PH, Goldberg BB, Mulholland SG. Ultrasonic detection of nonopaque renal calculi. Radiology 1978;127:233-237.

3. Edell S, Zegel H. Ultrasonic evaluation of renal calculi. Am J Roentgenol 1978;130:261-263.

4. Cook JH III, Lytton S. Intraoperative localization of renal calculi during nephrolithotomy by ultrasound scanning. J Urol 1977; 117:543-546.

5. Desai M, Ridhorkar V, Patel S, Bapat S, Desai M. Pediatric percutaneous nephrolithotomy: Assessing impact of technical innovations on safety and efficacy. J Endourol 1999; 13:359-364.

6. IMV. IMV 2006 CT Market Summary Report. Des Plains, IL: IMV Medical Information Division, 2006.

7. Ferrandino MN, Bagrodia A, Pierre SA, et al. Radiation exposure in the acute and short-term management of urolithiasis at 2 academic centers. J Urol 2009;181:668-673.

8. Ripolles T, Agramunt M, Errando J, Martinez MJ, Coronel B, Morales M. Suspected ureteral colic: Plain film and sonography vs unenhanced helical CT. A prospective study in 66 patients. Eur Radiol 2004;14:129-136.

9. Kartal M, Eray O, Erdogru T, Yilmaz S. Prospective validation of a current algorithm including bedside US performed by emergency physicians for patients with acute flank pain suspected for renal colic. Emerg Med J 2006; 23:341-344.

10. Smith-Bindman R, Aubin C, Bailitz J, et al. Ultrasonography versus computed tomography for suspected nephrolithiasis. N Engl J Med 2014;371:1100-1110. 
11. Türk C, Petrrík A, Sarica K, et al. EAU guidelines on diagnosis and conservative management of urolithiasis. Eur Urol 2016;69:468-474.

12. Tasian GE, Copelovitch L. Evaluation and medical management of kidney stones in children. J Urol 2014;192:1329-1336.

13. Semins MJ, Matlaga BR. Kidney stones during pregnancy. Nat Rev Urol 2014;11:163-168.

14. Passerotti C, Chow JS, Silva A, et al. Ultrasound versus computerized tomography for evaluating urolithiasis. J Urol 2009;182:1829-1834.

15. Patel SJ, Reede DL, Katz DS, et al. Imaging the pregnant patient for nonobstetric conditions: Algorithms and radiation dose considerations. Radiographics 2007;27:1705-1722.

16. Fulgham PF, Assimos DG, Pearle MS, et al. Clinical effectiveness protocols for imaging in the management of ureteral calculous disease: AUA technology assessment. J Urol 2012;189:1203-1213.

17. Kulkami NM, Pinho DF, Kambadakone AR, et al. Emerging technologies in CT-radiation dose reduction and dualenergy CT. Semin Roentgenol 2013;48:192-202.

18. Keilar AZ, Shabana W, Vakili M, et al. Prospective evaluation of Doppler sonography to detect the twinkling artifact versus unenhanced computed tomography for identifying urinary tract calculi. J Ultrasound Med 2012;31:1619-1625.

19. Abdel-Gawad M, Kadasne RD, Elsobkr E, Ali-El-Dein B, Monga M. A prospective comparative study of color Doppler ultrasound with twinkling and noncontrast computerized tomography in the evaluation of acute renal colic. J Urol 2016;196:1-6.

20. Ray AA, Ghiculete D, Pace KT, et al. Limitations to ultrasound in the detection and measurement of urinary tract calculi. Urology 2010;76:295-300.

21. Dunmire B, Lee FC, Hsi RS, et al. Tools to improve the accuracy of kidney stone sizing with ultrasound. J Endourol 2015;29:147-152.

22. Dunmire B, Harper JD, Cunitz BW, et al. Use of the acoustic shadow width to determine kidney stone size with ultrasound. J Urol 2016;195:171-177.

23. May PC, Haider Y, Dunmire B, et al. Stone-mode ultrasound for determining renal stone size. J Endourol 2016;30:958-962.

24. Ljunghall S, Danielson BG. A prospective study of renal stone recurrences. Br J Urol 1984;56:122-124.

25. Hyams ES, Shah O. Evaluation and follow-up of patients with urinary lithiasis: Minimizing radiation exposure. Curr Urol Rep 2010;11:80-86.

26. Mitterberger M, Pinggera GM, Pallwein L, et al. Plan abdominal radiography with transabdominal native tissue harmonic imaging ultrasonography vs unenhanced computed tomography in renal colic. BJU Int 2007;100:887-890.

27. Wickham JE, Kellett MJ. Percutaneous nephrolithotomy. Br J Urol 1981;53:297-299.

28. Lipkin ME, Mancini JG, Toncheva G, et al. Organ-specific radiation dose rates and effective dose rates during percutaneous nephrolithotomy. J Endourol 2012;26:439-443.

29. Mancini JG, Raymundo EM, Lipkin M, et al. Factors affecting patient radiation exposure during percutaneous nephrolithotomy. J Urol 2010;184:2373-2377.

30. Agarwal M, Agrawal MS, Jaiswal A, Kumar D, Yadav H, Lavania P. Safety and efficacy of ultrasonography as an adjunct to fluoroscopy for renal access in percutaneous nephrolithotomy (PCNL). BJU Int 2011;108:1346-1349.

31. Yan S, Xiang F, Yongsheng S. Percutaneous nephrolithotomy guided solely by ultrasonography: A 5-year study of $>700$ cases. BJU Int 2013;112:965-971.
32. Chi T, Masic S, Li J, Usawachintachit M. Ultrasound guidance for renal tract access and dilation reduces radiation exposure during percutaneous nephrolithotomy. Adv Urol 2016;2016:3840697.

33. Usawachintachit M, Masic S, Chang HC, Allen IE, Chi T. Ultrasound guidance to assist percutaneous nephrolithotomy reduces radiation exposure in obese patients. Urology 2016. [Epub ahead of print]; DOI: 10.1016/j.urology.2016.04.012.

34. Usawachintachit $\mathrm{M}$, Masic S, Allen IE, Li J, Chi $\mathrm{T}$. Adopting ultrasound guidance for prone percutaneous nephrolithotomy: Evaluating the learning curve for the experienced surgeon. J Endourol 2016;30:856-863.

35. Li X, Long Q, Chen X, He D, He H. Real-time ultrasoundguided PCNL using a novel SonixGPS needle tracking system. Urolithiasis 2014;42:341-346.

36. Li R, Li T, Qian X, Qi J, Wu D, Liu J. Real-time ultrasonography-guided percutaneous nephrolithotomy using SonixGPS navigation: Clinical experience and practice in a single center in China. J Endourol 2015;29:158-161.

37. Deters LA, Belanger G, Shah O, Pais VM. Ultrasound guided ureteroscopy in pregnancy. Clin Nephrol 2013;79:118-123.

38. Deters LA, Dagrosa LM, Herrick BW, Silas A, Pais VM Jr. Ultrasound guided ureteroscopy for the definitive management of ureteral stones: A randomized, controlled trial. J Urol 2014;192:1710-1713.

39. Harper JD, Cunitz BW, Dunmire B, et al. First in human clinical trial of ultrasonic propulsion of kidney stones. J Urol 2016;195:956-964.

40. Sorensen MD, Bailey MR, Shah AR, et al. Quantitative assessment of shockwave lithotripsy accuracy and the effect of respiratory motion. J Endourol 2012;26:1070-1074.

41. Chen CJ, Hsu HC, Chung WS, Yu HJ. Clinical experience with ultrasound-based real-time tracking lithotripsy in the single renal stone treatment. J Endourol 2009;23:1811-1815.

42. Abid, N, Ravier, E, Promeyrat, X, et al. Decreased radiation exposure and increased efficacy in extracorporeal lithotripsy using a new ultrasound stone locking system. J Endourol 2015;29:1263-1269.

\section{Address correspondence to: Thomas Chi, MD Department of Urology University of California, San Francisco 400 Parnassus Avenue Suite A610 Box 0330 San Francisco, CA 94143 \\ E-mail: tom.chi@ucsf.edu}

$\begin{aligned} & \text { Abbreviations Used } \\ \text { ALARA } & =\text { as low as reasonably achievable } \\ \text { AUA }=\text { American Urological Association } & \text { CDU }=\text { color Doppler ultrasound } \\ \mathrm{CT} & =\text { computed tomography } \\ \mathrm{EAU} & =\text { European Association of Urology } \\ \mathrm{ED} & =\text { emergency department } \\ \mathrm{KUB} & =\text { kidney, ureter, and bladder radiograph } \\ \mathrm{MRI} & =\text { magnetic resonance imaging } \\ \mathrm{NCCT} & =\text { noncontrast computed tomography } \\ \mathrm{PCNL} & =\text { percutaneous nephrolithotomy } \\ \text { POCUS } & =\text { point-of-care ultrasound } \\ \text { RIRS } & =\text { retrograde intra-renal surgery } \\ \mathrm{SWL} & =\text { extracorporeal shockwave lithotripsy }\end{aligned}$

